\title{
La toma de decisiones individuales \\ con riesgo desde la psicología
}

\author{
Orfelio G. León
}

Universidad Autónoma de Madrid

\section{INTRODUCCION}

En el presente trabajo tratamos de presentar algunas de las características de los estudios de toma de decisiones en psicología. En primer lugar es necesario acotar que nos referiremos a las decisiones individuales y además en ambiente de riesgo (con probabilidades conocidas), si bien muchas de las consideraciones que se harán son aplicables al resto de los ámbitos de la toma de decisiones.

Comenzaremos haciendo algunas consideraciones históricas respecto del origen de la toma de decisiones para a continuación pasar a centrar el tema con referencia a la psicología matemática y a la psicología cognitiva. En el punto siguiente nos detendremos brevemente sobre algunos de los tópicos de mayor relevancia en toma de decisiones (la tarea, el decisor y la respuesta). Continuaremos haciendo una discusión sobre las distintas formas de concebir los modelos en su aspecto más general, a saber: descriptivos versus normativos. Es obligado referirse siempre que se hace una reflexión sobre la teoría de la decisión a la aportación de lo que se ha venido en denominar teoría moderna de la utilidad (Von Neumann y Morgenstern, 1944), por lo tanto dedicaremos un breve espacio a comentar esta aportación. Como resultado de la progresiva psicologización de las teorías comportamentales de la toma de decisiones con riesgo presentamos a grandes rasgos la aproximación de Kahneman y Tversky (1979), para concluir con un intento de panorama integrador de los estudios de toma de decisiones.

\section{ORIGENES Y MARCO ACTUAL}

Existe un cierto consenso en los manuales de teoría de la decisión en referirse a los matemáticos franceses de los siglos XVII y XVIII como los iniciadores de los estudios de decisión al tratar de encontrar matemáticamente una pauta de comportamiento óptima en los juegos de azar, a petición de los nobles de la época. Se cita también comúnmente el año de 1738 y el nombre de Bernouilli como el primer arranque documentado del tema al que nos referimos.

A pesar de lo separado en el tiempo del trabajo de Bernouilli hay algunas características de su aportación que merecen ser destacadas. Es la primera vez que se introduce un concepto plenamente psicológico: la utilidad. Todos los estudios anteriores suponían implícitamente que el valor que el sujeto asignaba a una cantidad monetaria coincidía exactamente con ésta. Los modelos desarrollados bajo este presupuesto vinieron a denominarse de valor esperado, coincidiendo éste con la es- 
peranza matemática del juego. La solución propuesta por Bernouilli consistía en, una vez sustituido el valor por la utilidad, explicar la conducta del sujeto racional como la maximización de la utilidad esperada. Es importante notar que por primera vez, como ocurrirá luego a lo largo de la historia de la teoría de la decisión, se funden dos propósitos, por un lado se trata de describir de la mejor forma posible la conducta del sujeto racional (que sería el objetivo más psicológico, por decirlo así) y por otro lado se pretende deducir cuál es la mejor forma de decidir, suponiendo que se quiere obtener el máximo de beneficio. La segunda característica de mayor relevancia fue el establecimiento de la función de utilidad. Esta función asignaba un valor subjetivo a las cantidades monetarias tal que resultaban incrementos marginales decrecientes y por lo tanto la forma de esta función era cóncava. Aunque a lo largo del tiempo se han configurado distintas formas para la función de utilidad, el carácter cóncavo se ha mantenido, al menos para las ganancias.

El desarrollo de la teoría de la decisión, aunque ha encontrado un amplio campo de aplicaciones, se ha producido principalmente en tres áreas: la economía, las matemáticas y la psicología. En la economía principalmente dentro del área denominada microeconomía, con relación a los problemas de dirección de empresas y estudios de mercado en tanto en cuanto comportan decisiones de inversión y reparto de recursos por un lado, y por otro el conocimiento de los parámetros de la conducta del consumidor. Las matemáticas han estudiado, bajo el mismo título de teoría de la decisión o análisis de decisiones, los problemas generales desde un puesto de vista formal, proporcionando, a partir del cumplimiento de un conjunto de axiomas, la justificación de la existencia de una determinada función de utilidad. La psicología, en la medida en que las decisiones implicaban un decisor humano, ha ido incorporando los conocimientos sobre los procesos mentales superiores relacionados con la toma de decisiones. Las aplicaciones que desde estos tres campos se han llevado a cabo abarcan la medicina, el marketing, la ingeniería, la política..., de hecho desde hace unos años vienen funcionando empresas privadas que ofrecen servicios para ayuda a la decisión.

Los primeros psicólogos que se dedican a estudiar la teoría de la decisión son los que se encuadran dentro de la psicología matemática, posiblemente por el carácter formal de sus procedimientos surgidos en la economía y en las matemáticas. Desde finales de los años cincuenta se convierte en un tema clásico de la psicología matemática y empieza a aparecer como un capítulo propio en los manuales de este área (Luce, Bush y Galanter, 1965; Coombs, Daws y Tversky, 1970; Krantz, Atkinson, Luce y Suppes, 1974). Un indicador de la importancia que ha ido adquiriendo el tema en la psicología puede ser el comentario que realizan Slovic, Fischoff y Lichtenstein (1977) en su revisión del tema, cifrando el volumen de referencias relevantes sobre decisión, solamente en el periodo de 1971 a 1975 , en más de mil.

Sin embargo nosotros pensamos que gran parte de la resonancia de este tema se debe al hecho de haber sido permeable a los enfoques preponderantes en la psicología actual. Coombs y Lehner (1984) recogen esta idea y afirman que la mayoría de las investigaciones actuales en teoría de la decisión (en psicología) son consideraciones cognitivas en el procesamiento de la información. Esta proximidad con la psico- 
sino que los propios cognitivos lo han considerado así y podemos encontrarnos con capítulos dedicados a la toma de decisiones en manuales clásicos de psicología cognitiva como el de Anderson (1972) o el de Lindsay y Norman (1977).

Con el propósito de materializar estas supuestas relaciones, nos proponemos presentar a continuación un análisis donde se pretenden establecer unas caracterísiticas similares y comunes a ambos desarrollos psicológicos. En primer lugar presentamos cuatro cualificadores del origen y desarrollo de la psicología cognitiva y la teoría psicológica de la decisión.

Del conjunto de factores que se suelen citar en los libros generales de psicología cognitiva (Delclaux y Seoane, 1982; De Vega, 1984) podemos extraer los siguientes: 1) La psicología cognitiva se caracteriza por tomar prestados, en sus orígenes, problemas o métodos de trabajo de otras ciencias; como es el caso de la lingüística o la informática. En la teoría de la decisión podemos observar que tanto el objeto como el método es transvasado desde la economía y las matemáticas. Observemos que el título de la obra de Von Neuman y Morgenstern (1944) es Theory of games and economic behavior. 2) Se puede caracterizar también la psicología cognitiva por el esfuerzo en superar el reduccionismo del esquema estímulo-respuesta y tratar en consecuencia de hacer objeto de la psicología precisamente aquello que quedaba fuera en el conductismo. En la teoría psicológica de la decisión, Kozielecki (1981), señala como característico el propósito de superar el marco reduccionista de tarea-elección con el que se trabajaba en los orígenes matemáticos de la teoría, preocupándose especialmente por los fenómenos no abordados en este enfoque, es decir el procesamiento de información que precede a la decisión. 3) Progresiva influencia del enfoque del procesamiento de la información, en un sentido débil, en cuanto al uso de la metáfora del ordenador y las posibilidades que el lenguaje de los diagramas de flujo puede aportar a la comprensión de los procesos mentales superiores. La idea de concebir al decisor humano como un procesador activo de información y, por lo tanto, participar de las aportaciones del enfoque del procesamiento de información se puede detectar en la revisión de Einhorn y Hogarth (1981) sobre toma de decisiones. 4) La preocupación por las actividades mentales superiores ha potenciado la investigación sobre temas como el pensamiento o el razonamiento, anteriormente inabordables desde el paradigma conductista (Carretero y García-Madruga, 1984). La existencia de sesgos y heurísticos en el razonamiento humano se ha puesto de manifiesto como característico de este tipo de procesos. Por otro lado, en decisión, los últimos modelos han tratado precisamente de incorporar estos sesgos, cuya existencia era conocida desde los primeros estudios, pero no era posible dar cuenta de ellos desde modelos de razonamiento estrictamente lógicos. (Véase Kahneman y Tversky, 1979.)

Por último, pensamos que algunos de los juicios críticos sobre el estado actual de la psicología cognitiva pueden ser pertinentemente aplicados a la teoría psicológica de la decisión. La proliferación de micromodelos y la falta de base globalizadora han sido puestas de manifiesto por diversos autores, entre otros Marx y Goodson (1976). Igualmente Krantz el al. (1974) llamaban la atención sobre la excesiva acumulación de datos y la falta de principios básicos consensuados que guíen la conducta preferencial humana. Demasiado a menudo, en el momento presente de la psicología, da la sensación que todos los experi- 
mentos confirman las hipótesis experimentales y de que al mismo tiempo la realidad se amolda contradictoriamente a explicaciones incompatibles. Como señalaba Delclaux (1981), la autocensura de los investigadores y de las redacciones de publicaciones científicas impone la no publicación de muchos resultados experimentales donde no se puede rechazar la hipótesis nula. Esto produce un sesgo evidente al tratar de integrar la información. En segundo lugar, a pesar de que cada vez más se trata de corregir, existe una falta de integración sistémica entre los procesos mentales superiores: atención, percepción y memoria; esto repercute directamente sobre la toma de decisiones, ya que está directamente ligada a los procesos mencionados. En tercer y último lugar podemos decir que tanto la psicología cognitiva como la teoría de la decisión han heredado, afortunadamente a nuestro modo de ver, el refinamiento experimental desarrollado durante los años de preponderancia del conductismo.

\section{PRINCIPALES ELEMENTOS DE LA TEORIA PSICOLOGICA DE LA DECISION}

Como ya apuntábamos en la introducción, dedicaremos este punto a tres de los elementos principales de la teoría psicológica de la decisión: la tarea, el decisor y la respuesta. Estos serían longitudinalmente los tres puntos que recorre toda decisión y que en términos de procesamiento de la información corresponderían a la entrada, el procesamiento y la salida.

La tarea fue considerada desde el comienzo de los estudios de decisión como el primer y principal determinante de la elección. Si bien hoy no se mantiene que sea el más importante si se sigue considerando que su conocimiento es básico para poder entender la decisión. Hasta tal punto es crucial que, ante la falta de generalización de los patrones decisorios observados, parece que cada vez más se tiende a explicar las decisiones acotadas para un determinado conjunto de tareas de características comunes.

La necesidad de diferenciar las tareas ha llevado a proponer distintos criterios de clasificación. Por ejemplo, Howard (1968), propuso un espacio tridimensional para su ubicación, contando con las siguientes dimensiones: $a$ ) complejidad, $b$ ) dinamismo, $c$ ) riesgo. El eje de complejidad evaluaría el grado de dificultad objetivo para realizar la tarea. Independientemente de los contenidos, el número de opciones, la cantidad de dimensiones incluidas en cada opción y el hecho de que éstas sean simples o compuestas, pueden ser elementos para evaluar el grado de complejidad de la tarea. El dinamismo hace referencia al desarrollo temporal, por lo tanto ésto permite categorizar a las tareas de estáticas hacia dinámicas. En último lugar el riesgo evalúa cómo de seguro es el conocimiento que el sujeto tiene de los elementos implicados, pudiendo dicotomizarse las tareas en deterministas y probabilistas.

Además de las razones expuestas al comienzo de este párrafo, para un psicólogo, existen dos aspectos tremendamente importantes que deben ser evaluados en las tareas. En primer lugar el papel que ésta desempeña como determinante del espacio de la tarea (en términos de $\mathrm{Ne}$ well y Simon, 1972) ya que el decisor humano trabajaría precisamente sobre este espacio en su evaluación y resolución. En segundo lugar habrá que evaluar el grado de generalización de la conducta, si se quiere, 
el valor ecológico que la situación experimental tiene respecto de la vida cotidiana de los seres humanos. Estas cuestiones, que para una aproximación formal son irrelevantes, se convierten en cruciales para la construcción del conocimiento psicológico.

El decisor, que en los orígenes matemáticos de la teoría era un elemento pasivo de la lógica y cuyo comportamiento venía dirigido exclusivamente por las características de la tarea, ha ido tomando cada vez más protagonismo a medida que el enfoque se hacía más psicológico. Como decíamos en párrafos anteriores, da concepción actual del decisor humano como un procesador activo de información, no sólo le ha dado más relevancia a su papel dentro del proceso de la toma de decisiones, sino que le ha convertido en el pivote central de la teoría.

Si enmarcamos la decisión en una estructura más básica que la posibilite, deberemos tener en cuenta (Kozielecki, 1981) que toda tóma de decisión implica de una manera u otra el logro de un objetivo, el uso de sistemas de almacenamiento de información y una determinada seriación del procesamiento. En primer lugar en tanto en cuanto se propone conseguir un objetivo, la toma de decisiones deberíamos verla como un caso particular de logro de metas y por lo tanto pensar que se halla estructurada por los mismos factores que son descritos por Newell y Simon (1972) para el caso general, es decir: la utilización de medios, la descomposición en subobjetivos, la existencia de un criterio de continuación, la evitación de repeticiones, y la existencia de un criterio de finalización. Estas características, habitualmente contrastadas en los estudios de resolución de problemas, parece claro que deberían ser cumplidas por cualquier programa de simulación de toma de decisiones.

Las limitaciones del procesador humano, en cuanto a los sistemas de almacenamiento de la información que se tienen que usar en el proceso de la decisión, es el segundo punto básico que se tiene que evaluar. Los tempranos estudios sobre la limitación de la memoria de trabajo, dentro de la cual se lleva a cabo el proceso que nos ocupa, han preocupado a los estudiosos de la decisión aplicada, ya que esto parecería suponer un grave inconveniente para la capacidad decisoria de los seres humanos sin ayuda externa. En concreto, los trabajos de Elstein y Bordage (1979) sobre el número máximo de diagnósticos que podían contrastarse simultáneamente revelaron que se hallaba en cinco más menos dos. Por otro lado, las peculiaridades del almacenamiento, clasificación y recuperación de la memoria a largo plazo están directamente implicadas en la utilización de estrategias aprendidas o en el uso de «bancos de datos». Los heurísticos de representatividad y accesibilidad estarían precisamente posibilitados por estas condiciones.

El último punto de enmarcamiento estructural es la forma de seriación en el procesamiento. Ciertamente no se postula una forma de procesamiento típica de la toma de decisiones, aunque parece considerarse que dada la dificultad de las tareas, incluso las más sencillas, la toma de decisiones se tendrá que realizar de una forma serial. Lopes y Ekberg (1980) y León y Botella (1985), utilizando el tiempo de latencia como respuesta, han contrastado hipótesis sobre la ordenación en el procesamiento del heurístico de anclaje y ajuste, asumiendo en ambos casos un procesamiento serial.

La respuesta, el tercero de los elementos que nos habíamos propuesto comentar, se caracteriza por una enorme diversidad de modos y por la no generalización de los resultados, para estructuras de decisión similares. La forma de respuesta más usada es la de verbalizar las 
preferencias entre opciones. Este fue el método que utilizó el matemático francés Allais (1953) cuando realizó la primera comprobación de los axiomas de la teoría de la utilidad esperada y sigue apareciendo en los experimentos actuales, como se puede comprobar en el libro de Wright (1985). Mosteller y Noguee (1951) les daban a los sujetos una cantidad de dinero para que pudieran jugar, asumiendo por tanto los resultados de sus decisiones. Si bien este procedimiento es más ecológico, en términos actuales, hace que el estado de riqueza inicial no sea cero y por lo tanto el comportamiento de los sujetos será más bien equivalente a una situación en la que en la primera jugada hubieran ganado la cantidad que los experimentadores les daban. Tversky (1967) medía la respuesta de los sujetos pidiéndoles que estableciesen el precio mínimo por el que estarían dispuestos a vender una opción probabilística. Anderson y Shanteau (1970) pedían a sus sujetos experimentales que valorasen una opción (probabilidad y valor) marcando una distancia en una recta, tal que el punto extremo de la izquierda representaba no ganar nada y el punto extremo de la derecha representaba ganar con toda seguridad el más alto de los valores que aparecían en el conjunto. Una dificultad de este procedimiento es que los sujetos pueden llevar a sus extremos el sesgo de anclaje y a juste dando algún valor a opciones con premios muy altos, pero con probabilidad nula (León, Rueda y Vega, en prensa). Otra de las formas utilizadas (Lichtenstein y Slovic, 1971) ha sido la de pedirle al sujeto que realice apuestas monetarias sobre cada una de las opciones que se le presentan, en función del valor que él asigna a cada una de ellas. El tiempo de latencia en la elección fue utilizado por Lopes y Ekberg (1980).

Se han desarrollado también algunas formas de medir las elecciones especialmente adecuadas para el tipo de tarea en estudio, como es el caso del experimento de Ebbensen et al. (1977), donde se estudiaba el comportamiento de los conductores ante la situación de tener que cruzar en un stop, y por lo tanto tener que evaluar si las dimensiones de distancia y velocidad a la que percibían un vehículo eran adecuadas para cruzar o no cruzar. La forma de respuesta utilizada en esta ocasión consistió en hacer que el sujeto cruzase con un móvil accionado por él en una maqueta construida al efecto y donde el experimentador manipulaba las condiciones de distancia y velocidad de un segundo móvil.

Aparte de mostrar una selección de los tipos de respuesta utilizados en la experimentación con toma de decisiones creemos que es importante poner de manifiesto el hecho de la no coincidencia de las formas de respuesta entre los diversos experimentadores, ya que este fenómeno se muestra revelador cuando comprobamos que los datos obtenidos por unos no se ajustan a los modelos utilizados por otros. Payne (1985), resumiendo datos recogidos en la literatura hasta ese momento, expone que de hecho las preferencias de los sujetos resultan invertidas cuando se modifica el tipo de respuesta utilizada. Estos resultados vienen a confirmar la necesidad de observar cómo el sujeto percibe, representa la tarea, valora, y combina los resultados de las fases implicadas en la elección, ya que leves modificaciones en las condiciones de la tarea o de la respuesta parecen alterar sus preferencias. Para poder acceder a este tipo de conocimiento, Simon (Ericson y Simon, $1980,1985)$ mantiene que se debe recoger la conducta verbal del sujeto durante el proceso de elección, de la misma forma que se viene haciendo para estudiar la resolución de problemas. 


\section{LOS MODELOS: DESCRIPTIVOS VS NORMATIVOS}

Nos ha parecido más interesante, a la hora de hablar de los modelos sobre decisión individual con riesgo, más que hacer una enumeración, que por su volumen se haría necesariamente tediosa y que se puede encontrar en las últimas revisiones aparecidas sobre el tema, centrar la discusión sobre un aspecto, pensamos que polémico, que no sólo pretende dividir los tipos de modelos, sino la forma de investigar.

Desde Von Neuman y Morgenstern se viene hablando de modelos descriptivos y modelos normativos. Sin embargo, o bien se ha dicho que los modelos cumplían ambos propósitos, como fue el caso de la teoría de la utilidad o bien se concluía que en la práctica era casi imposible mantener esta división. Arce (1982) concluye de esta manera cuando introduce su trabajo de clasificación de los modelos y de hecho este criterio no aparece en el encuadre posterior. Esta posición se ha venido manteniendo hasta Pitz y Sachs (1984). Nosotros también pensamos que podrían existir algunos criterios diferenciadores, no sólo como decíamos de los tipos de modelos, sino de la forma de plantear las investigaciones. Los puntos discriminantes serían los siguientes:

1) Serían modelos normativos aquellos que fundamentalmente se propusieran construir algoritmos que optimizasen las decisiones de los humanos, independientemente de cómo éstos las realicen espontáneamente. Mientras que serían modelos descriptivos aquellos que se propusiesen explicar cómo los seres humanos toman las decisiones, independientemente de si estas decisiones son óptimas o no.

2) Los trabajos normativos estarían interesados principalmente por un análisis formal de la tarea; en este análisis se fundamentará el cuerpo axiomático del modelo. Los trabajos descriptivos, además del análisis formal de la tarea, estarían interesados primordialmente en la representación que el sujeto se hace de la tarea, ya que esta representación no es necesariamente isomórfica y además será sobre esta representación sobre la que el sujeto elaborará su elección.

3) La forma implícita de razonamiento del decisor en los modelos formales viene explicada por la lógica formal, es decir la lógica que utilizan las matemáticas para deducir de un conjunto de axiomas una regla de preferencias. Las aproximaciones descriptivas, siguiendo el comportamiento real de razonamiento de los sujetos, incorporan aquellos sesgos y heurísticos de los que se tiene evidencia que conforman el razonamiento humano y que desde luego no se ajustan a los patrones de la lógica formal.

4) Los modelos formales definen situaciones de decisión que se pueden denotar por una gran simplificación de las variables humanas intervinientes, apenas un conjunto de preferencias sobre resultados simples, mientras que los modelos descriptivos tratan de incluir, porque teóricamente no se puede entender un aspecto de la conducta de forma aislada, el máximo de complejidad cuyo comportamiento regular pueda ser incluido en un modelo.

5) Por último la forma y el momento en que se utiliza la experimentación, pensamos que puede discriminar una forma u otra de las que venimos hablando. En los modelos más matemáticos, la experimentación se utiliza una vez que se ha completado la teoría y se disponen de predicciones directamente contrastables con la realidad, mientras que los datos empíricos, durante el proceso de elaboración del modelo, sólo sirven para inferir qué axiomas pueden ser más viables. Las aproximaciones más psicológicas utilizan la experimentación para inducir cuáles deberían ser los parámetros y cuál su aportación en el modelo. Salvando las distancias sería una diferencia parecida a la que existió entre los trabajos de Fechner y Stevens. Una vez construido el modelo igualmente se deducirán hipótesis contrastables para falsearlo. 
Estas cinco características son cumplidas de forma exclusiva, por una parte por la teoría de la utilidad (normativa) y por la otra por la teoría prospectiva (descriptiva). Ciertamente los modelos existentes no pueden ser categorizados (ni lo pretendíamos) en dos conjuntos disjuntos, pero sí pueden servirnos para denotar cómo de cerca se halla cada trabajo de cada uno de los dos enfoques propuestos. En los dos puntos siguientes comentaremos algunas características de los modelos citados de Von Neuman y Morgenstern (1944) y de Kahneman y Tversky (1979), para tratar de clarificar sobre las propias teorías las diferencias discutidas en este apartado.

\section{TEORIA DE LA UTILIDAD ESPERADA}

La importancia que ha tenido la teoria moderna de la utilidad esperada hace que siempre que se hace una reflexión sobre la toma de decisiones con riesgo, en mayor o menor medida, se dedique un espacio a considerar la aportación de este enfoque. De una forma breve, podemos decir que si las preferencias de los sujetos satisfacen un conjunto de axiomas, entonces la conducta racional puede establecerse como la maximización de la utilidad esperada. Esta utilidad es asignada por medio de una función real, cuya existencia se demuestra directamente del cumplimiento de los axiomas.

Desde muy temprano se propusieron variaciones al conjunto de axiomas establecidos por Von Neuman y Morgenstern, como por ejemplo la muy difundida de Luce y Raiffa (1957). Esta última tiene el interés suplementario de ser llevada a cabo por dos autores que son encuadrados claramente como psicólogos matemáticos, cuyo trabajo en distintas facetas de este área ha tenido desde mediados de los años cincuenta hasta nuestros días una amplia resonancia. La variación fundamental de esta última axiomática citada fue la de considerar el conjunto de opciones finitas lo cual se traducía en una acotación de la función de utilidad, cosa que antes no ocurría. Los cuatro axiomas pasan a ser seis: 1) Existencia de una relación de orden débil en el conjunto de resultados. 2) Reducción de las opciones compuestas a simples. 3) Continuidad o mixtura. 4) Sustitución. 5) Existencia de una relación de orden débil en el conjunto de opciones compuestas. 6) Monotonía. La función de utilidad deducida del cumplimiento de estos axiomas resulta ser isótona, lineal y única. La función de utilidad permite (previa normalización de los valores de utilidad que establece un valor cero para el resultado menos preferido y un valor de uno para el más preferido) calcular, por el axioma de mixtura, cuál es real entre cero y uno que se le asigna a cada resultado. El valor de cada opción compuesta se calcula por la combinación lineal de probabilidades especificadas y los valores de utilidad de los resultados calculados, permitiéndonos decir que deberá ser preferida aquella opción cuyo valor de utilidad sea más alto.

Los trabajos críticos a este enfoque, especialmente desde la psicología han sido numerosos. Nosotros proponemos un agrupamiento, exclusivamente en orden a la mayor claridad en la presentación, en tres grandes bloques. Uno, las críticas al cumplimiento de los axiomas, otro, la igualdad implícita entre racionalidad humana y lógica formal y el último el papel de las probabilidades numéricas.

Con respecto al primer punto podemos decir que desde muy pronto aparecieron datos experimentales que cuestionaban el cumplimien- 
to de los axiomas por parte de las personas. Se suele citar como primer trabajo, cuyos resultados se han mantenido a lo largo del tiempo, el de Allais (1953). Otros datos sobre el incumplimiento de los axiomas se pueden encontrar en la obra citada de Coombs et al. (1970).

Desde el punto de vista psicológico nos parece más trascendente el segundo punto citado, ya que no sólo se daba por hecho el pensamiento racional humano lo era en tanto seguía las reglas de la lógica, sino que durante muchos años esto ni siquiera se contrastó. Una explicación desde un punto de vista de la historia de la ciencia la podríamos encontrar en el predominio del positivismo lógico, como marco epistemológico, que igualaba racionalidad con lógica formal. En el mismo Piaget, en otro contexto, podemos encontrar la similitud de pensamiento en la etapa de las operaciones formales con la conducta (lógica) del científico. Sin embargo, ya desde los años sesenta se empezó a cuestionar la generalidad de las características propias del periodo delas operaciones formales (Smedslund, 1963) y por lo tanto la validez del binomio racionalidad=lógica. Tversky y Kahneman (1974), recogiendo algunos trabajos de principios de los setenta, publicaron un artículo que luego ha pasado a ser un clásico de lo que se ha venido a denominar heurísticos y sesgos del pensamiento humano. Los principales heurísticos estudiados en el trabajo aludido son el de representatividad, accesibilidad, anclaje y ajuste, y sobreconfianza. Simon (1979), en otro aspecto de esta misma línea, contraponía al concepto de racionalidad lógica el de racionalidad limitada, como propia de los seres humanos, donde aparecerían pautas no asumibles sin contradicciones por un sistema lógico formal. Nisbett y Ross (1980) han sido quizá, fuera - del entorno de la toma de decisiones, los que más han divulgado en estos últimos años los heurísticos como estrategias propias del razonamiento humano. En áreas afines como el pensamiento y lenguaje, las posturas de autores como Johnson-Laird $(1982,1983)$ y Wason (1983) preconizan el abandono absoluto de estructuras lógico formales para explicar las relaciones de estos procesos. En definitiva parece que un modelo de decisión que se desarrolle exclusivamente sobre la lógica matemática no podrá dar cuenta de la toma de decisiones de los seres humanos.

El tercer aspecto crítico a que hacíamos referencia se centraba en las probabilidades cuantitativas incluidas en el aspecto de riesgo que comporta la teoría de la utilidad. Ya en el año 1954, Savage y posteriormente Edwards (1956) desarrollaron la más importante de las variaciones que del modelo de la utilidad esperada se han hecho: el modelo de la utilidad subjetiva esperada. Este modelo parte precisamente de la crítica a la forma de incluir las probabilidades en el cálculo de los valores esperados finales. El razonamiento es intuitivamente plausible: si las cantidades monetarias son consideradas por los sujetos, no por su valor real, sino por el valor que los sujetos les asignan, las probabilidades manifiestas serán integradas no por su exacto valor cuantitativo, sino por el valor subjetivo que cada uno asigne a los números. (Sería como decir que 1/2 para un optimista vale subjetivamente más que $1 / 2$ para un pesimista). Este modelo, más psicologizado, sigue construyéndose de la misma manera que su predecesor, es decir, se parte de un conjunto de axiomas tales, que de su cumplimiento se puede derivar la existencia de sendas funciones de utilidad y probabilidad subjetiva. Esta ha sido, sin lugar a dudas, la aproximación más fructífera de este área, como se puede detectar en el hecho de que sigan apare- 
ciendo experimentos cuyos datos se ajusten bien al modelo (Arce, 1985). Sin embargo la proliferación de trabajos no se ha visto acompañada por el consenso en las conclusiones obtenidas. Esta gran acumulación de resultados contradictorios ha preocupado y preocupa a los estudiosos del tema, que no han dado con una explicación totalmente satisfactoria de la cuestión. Una de las razones aducidas que goza de mayor predicamento es la expuesta por Rapoport y Walsten (1970) en su revisión del tema: las variaciones en los resultados se deben a la variación de las tareas utilizadas por los experimentadores en su contrastación. Esta explicación estaría en la línea de lo discutido aquí al tratar el punto de la tarea, sin embargo no parece eludible buscar una segunda explicación en orden a delimitar qué características de las tareas son las que propician los cambios contradictorios respecto del modelo. Por último, tal y como se desprende de algunos trabajos teóricos, como el realizado por Shoemaker (1982), los numerosos modelos derivados de la teoría de la utilidad esperada y los trabajos experimentales desarrollados la convierten en el más poderoso heurístico de producción investigadora en este área hasta nuestros días.

\section{TEORIA PROSPECTIVA}

Esta teoría fue desarrollada por Kahneman y Tversky (1979) tratando de formular un modelo alternativo a la teoría de la utilidad subjetiva esperada, que pudiera incorporar en sus predicciones algunas de las situaciones conocidas en que las suposiciones de la teoría clásica eran violadas. (La traducción del artículo original se presenta en este dossier.)

Hay dos consideraciones previas que quisiéramos hacer sobre esta teoría. La primera de ellas es hacer notar que sus autores al comienzo del artículo, a modo de declaración de intenciones, dicen que tratan de proponer un modelo descriptivo. Resulta totalmente pertinente constatar que para este caso, como decíamos, es aplicable el criterio propuesto de diferenciación entre normativos y descriptivos (tratan de explicar lo mejor posible la conducta de los decisores, analizan la representación que el sujeto se hace de la tarea, incorporan algunos sesgos de razonamiento, se incluyen el máximo de variables y utilizan la experimentación previa a la construcción del modelo). La segunda consideración previa hace referencia a la revista donde publican su trabajo: Econométrica. A nuestro modesto entender esto quiere decir que a la teoría económica le interesa cuanto se pueda decir de los parámetros que rigen la conducta de toma de decisiones de los humanos; de esta forma se cierra de nuevo el círculo abierto en la economía cuando los psicólogos se acercan interesados por lo que de psicológico pueda tener la conducta económica. El enfoque normativo y el descriptivo estan llamados a interactuar.

A modo de introducción, presentan los autores una recopilación de sucesos donde el modelo clásico no predice correctamente los resultados. Entre estos efectos podemos destacar el efecto de certidumbre, por el cual un suceso con seguridad absoluta es sobrevalorado en comparación con sucesos probables. El efecto de aislamiento pone de evidencia que, contra lo que cabría esperar, las decisiones no son independientes del proceso de descomposición intermedio, aunque las probabilidades finales sean las mismas. El efecto reflejo consiste en que las preferen- 
cias son invertidas cuando a todos los resultados les restamos una cantidad similar tal que todos, siendo antes ganancias, se convierten en pérdidas. Todos estos efectos han sido contrastados experimentalmente y presentan los datos obtenidos.

El proceso de decisión, según los autores, consta de dos fases: la primera, de preparación y la segunda de valoración. En la preparación, la información objetiva suministrada por el medio es representada no de una manera fiel, sino con simplificaciones y modificaciones que hacen sea procesada más fácilmente y quede preparada, por decirlo así, para la siguiente fase. En la segunda se valora el impacto subjetivo de la probabilidad así como la utilidad de la recompensa.

A pesar de incorporar plausiblemente algunos de los efectos de sesgo en la toma de decisiones, según nuestra modesta opinión se le podrían formular algunas críticas que nosotros hemos delimitado en cuatro puntos: 1) En las tareas presentadas a los sujetos sólo se utiliza como modo de respuesta la preferencia verbal, cuando existen fundadas dudas sobre el comportamiento real de los sujetos cuando reciben información de sus decisiones (por ejemplo León y Gámbara, en prensa). 2) Para una teoría que pretende ser alternativa a la USE nos parece que se deberían analizar con el nuevo modelo los datos de experimentos anteriores y mostrar un mejor ajuste predictivo, cosa que no ha aparecido todavía. 3) Si bien los autores justifican la separación mostrando que los datos se ajustan mejor haciendo uso de esta propiedad, nos parece que para un modelo que pretende ser explicativo, debe justificarse un paramorfismo entre las operaciones mentales implicadas y las operaciones del modelo. 4) A pesar de que ellos indican que su modelo puede generalizarse a otras situaciones de decisión donde no se trabaje con cantidades monetarias y probabilidades numéricas, haría falta mostrar experimentalmente que esto ocurre así.

\section{PANORAMA INTEGRADOR}

Para concluir estas reflexiones sobre la toma de decisiones individuales con riesgo nos hemos propuesto trazar un presunto panorama integrador que relacione y dé sentido a las distintas aproximaciones al tema. Con este objeto presentamos a continuación el cuadro siguiente:

En primer lugar establecemos un gran conjunto denominado decisión conductual donde estarían incluidos todos los trabajos que de una manera u otra tengan como objeto de estudio la toma de decisiones humanas. Este gran conjunto estaría alimentado fundamentalmente por dos fuentes, por un lado las disciplinas estrictamente formales (matemáticas y economía) y por otro la psicología del pensamiento y psicología cognitiva (incluyendo principalmente los estudios sobre memoria, atención y resolución de problemas). De la interacción de ambas fuentes y según por cual esté más determinada aparecerían dos grandes grupos de modelos de la decisión: óptimos (formales) y heurísticos (descriptivos). Podemos hacer referencia aquí a los criterios establecidos en el apartado en que diferenciábamos ambos enfoques, y de manera sucinta podemos resumir en maximización de la utilidad por parte de los óptimos y explicación de la conducta por parte de los heurísticos, como objetivo primordial y como causas que determinan principalmente la configuración de los modelos, las consecuencias, en los primeros y los procesos mentales en los segundos. Las derivaciones que 


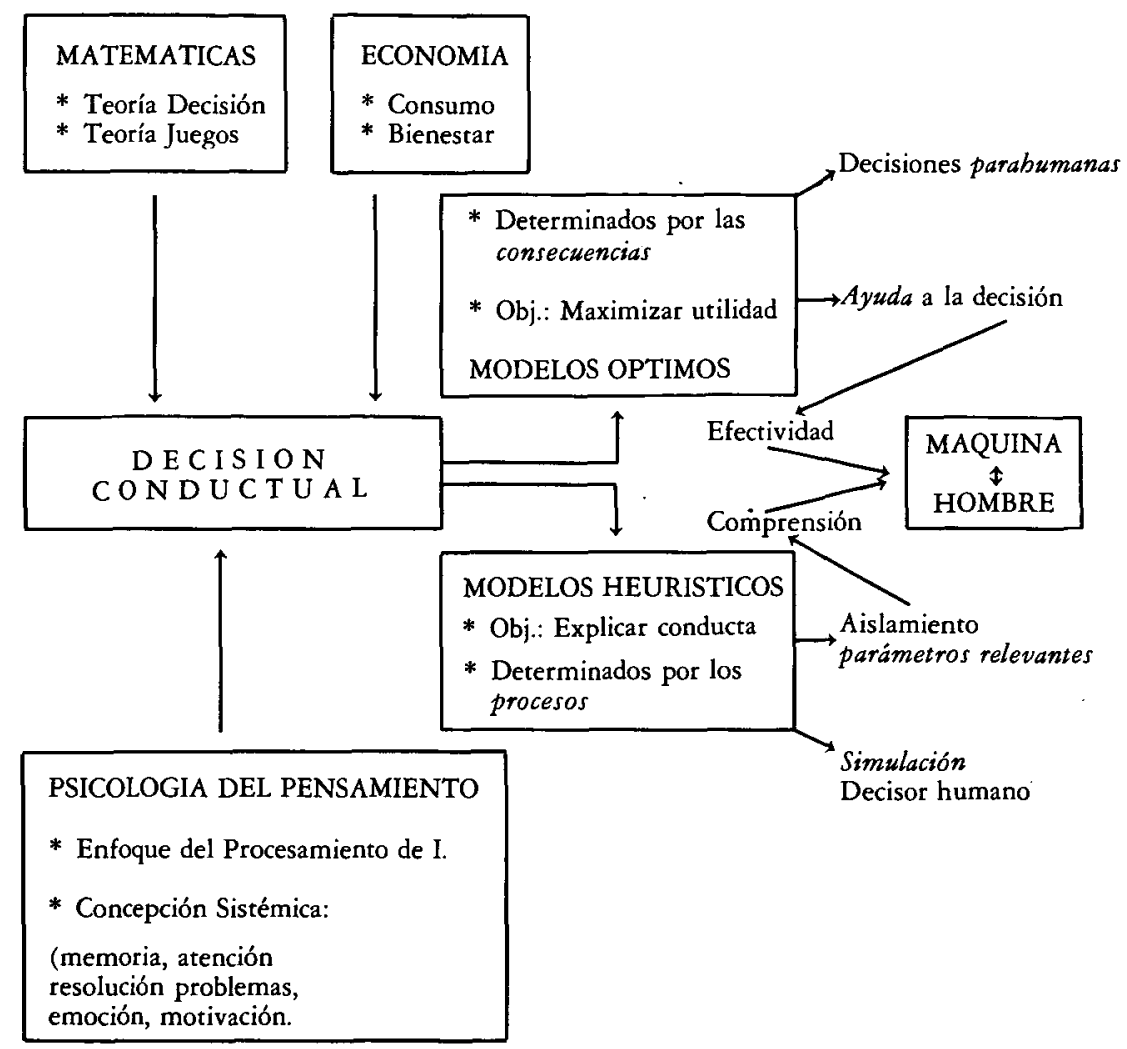

se originarían de ambos grupos serían, principalmente, el establecimiento de algoritmos para la ayuda a la decisión y por lo tanto pautas para las decisiones sociales y por el lado descriptivo la determinación de los parámetros que explican cómo en la práctica toman los seres humanos sus decisiones y por lo tanto cuáles deberían ser las guías de la simulación de decisiones.

Ambos tipos de trabajos, aparentemente divergentes, confluyen en el acto de la utilización de los programas de ayuda a la decisión por personas concretas. Esta relación la podríamos esquematizar como una relación hombre-máquina, donde para obtener unos resultados satisfactorios es necesario que los algoritmos implementados en los ordenadores cumplan con su objetivo de efectividad, pero al mismo tiempo se necesita de un especialista en decisión que haga comprensiva la utilización para la persona concreta que pretende servirse de la ayuda y que le proporcione la información necesaria sobre el comportamiento espontáneo de los seres humanos en toma de decisiones y en consecuencia los naturales sesgos que el razonamiento produce y cómo, contra su intuición, el programa le proporcionará una regla de decisión que optimice sus intereses explícitos. De esta forma volvemos a cerrar la línea abierta en un principio sobre las diferentes formas de abordar el estudio de la toma de decisiones, encontrando un punto donde tanto las aproximaciones formales como las descriptivas se ponen al servicio del ser humano. 


\section{Referencias}

Allais, M. «Le Comportement de L'Homme Devant le Risque: Critique des Postulats et Axiomas de L'Ecole Americaine». Econometrica, 1953, 21, 503-546.

ANDERSON, B. F. Cognitive Psychology: the Study of Knowing, Learning and Thinking. N. Y.: Academic Press, 1972.

ANDERSON, N., H. y ShanteaU, J. C. «Information Integracion in Risky Decision Making». Journal of Experimental Psychology, 1970, 84, 441-451.

ARCE, C.: Cambio en la preferencia por el riesgo en situaciones de dilemas de elección: Un estudio desde la perspectiva de la teoria de la utilidad. Universidad de Santiago de Compostela. Tesis sin publicar, 1982.

ARCE, C. «Polarización de Grupo, Decisiones Individuales y Utilidad Subjetiva Esperada en Situaciones de Elección bajo Riesgo: Un Estudio Experimental». Rev. Psicol. Gral. y Apl., 1985, 40 (1), 51-70.

Carretero, M., y Garcia Madruga, J. A. Lecturas de Psicología del Pensamiento. Madrid: Alianza Psicología, 1984 .

CoOmbs, C. H.; Dawes, R. M., y Tversky, A. Mathematical Psychology: An Elementary Introduction. Englewood Cliffs, N. Y.: Prentice-Hall. 1970. (Trad. Alianza Textos, 1980.)

COOMBS, C., H. y LeHNER, P. E. «Conjoint Desing and Analysis of the Bilinear Model: An Application to Judgments of Risk». J. of Math. Psychol., 1984, 28 (1): 1-43.

DELCLAUX, I. Inducción Probabilística y Explicación Psicologica Análisis y Modificación de Conducta, 1980, $6,139-145$.

Delclaux, I., y Seonne, J. Psicología Cognitiva y Procesamiento de la Información. Madrid: Pirámide, 1982.

EBBSEN, E. B.; PARKeR, S., y KONECNI, V. J. «Laboratory and Field Analysis of Decisions Involving Risk». Human Perception and Performance, 1977, 3 (4), 576-589.

Edwards, W. «The Theory of Decison Makin», Psychol. Bull., 1954, 380-417.

Einhorn, H. J., y Hogarth, R. M. «Behavioral Decision Theory: Processes of Judgment and Choice. Ann». Rev. Psychol., 1981, 32, 53-88.

ElsteIn, A. S., y BoRDAGE, G. «Psychology of Clinical Reasoning», en Stone y Alder (Eds.): Health Psychology. S. F.: Jossey-Bass, 1979.

Ericsson, K. A., y Simon, H. A. «Verbal Reports as Data». Psychol. Rev., 1980, 87, 215-251.

HowARD, R. A., «The Foundations of Decision Analysis». IEEE Transactions on Systems Science and Cybernetics, 1968, 4, 211-219.

Johson-LaIRD, P. N. Mental Models. Towards a Cognitive Science on Langiage, Inference, and Conciusness. Cambridge: Cambridge U. P., 1983.

Kahneman, D., y TVersky, A. «Prospecto Theory». Econometrica, 1979, 2, 263-291.

KozIELECKI, J. Psychological Decision Theory. Theory and Decision Library, 1981.

KRANTZ, D. H.; ATKINSON, R. C.; LUCE, R. D., y SUPPES, P. (Eds.): Contemporary Developments in Matbematical Psychology. Vol. 1. S. Francisco. Freeman, 1974.

LEON, O. G., y BOTELLA, J. "Sobre la seriación Ordenada del Anclaje y Ajuste: Una Réplica a Lopes y Ekberg». Boletin de Psicología, 1985, 8, 101-116.

León, O. G., y GÁmbara, H. «Relevancia de la Valoración de las Consecuencias en la Toma de Decisiones». Rev. Psi,ol. Gral. y Apli. (en prensa).

LEÓN, O. G.; RUEDA, R., y VEGA, A. «Personalidad, Diferencias Individuales y Decisión con Riesgo». Es tudios de Psicología (en prensa).

Lichtenstein, S. C., y Slovic, P. «Reversal of Preference between Bids and Choices in Gambling Decision». J. E. P., 1971, 89: 46-55.

Lindsay, P. H., y Norman, D. A. Human Information Processing (Introducción a la Psicología Cognitiva. Madrid: Tecnos, 1983). N. Y.: Academic Press, 1977.

LOPES, L. L., y EKBERG, P. H. S. «Test of an Ordering Hypothesis in Risky decision Making». Acta Psychologica, 1980, Vol. 45 (1-3), 161-167.

LOPES, L. «Decision Making in Short Run». J. of E. Psy.: Human Learning and Memory, 1981, 7 (5), 377-385.

LuCE, R. D.; Bush, R. R., y GaLANTeR, E. (Eds.): Handbook of Mathematical Psychology (3 vols.). N. Y.: Wiley, 1963.

LUCE, R. D., y RAIFFA, H. Games and Decisions. N. Y.: Wiley, 1957.

MARX, M. H., y Goodson, F. E. Theories in Contemporary Psychology. N. Y.: Macmillan, 1976.

Mosteller, F., y NOGEE, P. «An Experimental Measurement of Utility». J. of Political Economy, 1951, 371-404.

Newell, A., y Simon, H. A. Human Problem Solving. N. J.: Prentice Hall, 1972.

NisBeTT, R. E., y Ross, L. Human Inference: Strategies and Shortcomings of Social Judgment. N. J.: Prentice-Hall, 1980.

PAYNE, J. W. «Psychology Risky Decision», en G. Wright: Bebavioral Decision Making. N. Y.: Plenum, 1985 . 
PITZ, G. F., y SACHS, N. J. «Judgment and Decision: Theory and Decision and Application». Ann Rev. Psychol. 1984, 35, 139-163.

RAPOPORT, A., y WallsteIN, T. S. «Individual Choice Behavior». Ann Rev. Psychol., 1972, 23, 131-176. SavaGe, L. J. The Foundations of Statistics. N. Y.: Wiley, 1954.

ShoEmaker, P. J. H. «The Expected Utility Model: Its Variants Purposes, Evidence and Limitations». J. of Economic Literature, 1982, 20, 529-563.

Simon, H. A. «Rationality as Process and as Producto of Thought» An. Econ. Rev., 1979, 68, 1-16.

Slovic, P.; FisChOfF, B., y Lichtenstein, S. «Behavioral Decision Theory». Ann. Rev. of Psychol., 1977.

SMEDSLUND, J. «The Concept of Correlation in Adults». Scandinavian Journal of Psychology. 1963, 4, $165-173$.

TVERSKY, A. «Utility Theory and Additivity Analysis» J. of Exp. Psychol., 1967, 75, 27-37.

TVERSKY, A., y KAHNEMAN, D. «Judgment under Uncertainty: Heuristics and Biases». Science, 1974, 185, 1124-1131.

VEGA M. DE. Introducción a la Psicologia Cognitiva. Madrid: Alianza Psicología, 1984.

Von Neuman, J., y Morgenstern, O. Theory of Games and Economic Behavior. Princeton: Princeton U. P., 1944 .

WASON, P. «Realism and Rationality in the Selection Task», en J. Evans, (ed): Thinking and Reasoning. Psychological Approaches. Londres: Routledge and Kegan Paul, 1983.

WRIGHT, G. (Ed). Bebavioral Decision Making. N. Y.: Plenum Press, 1985. 